\title{
Biases in genome reconstruction from metagenomic data
}

\author{
William C Nelson $^{\text {Corresp., } 1}$, Benjamin J Tully ${ }^{2,3}$, Jennifer M Mobberley ${ }^{4}$ \\ ${ }^{1}$ Biological Sciences Division, Pacific Northwest National Laboratory, Richland, Washington, USA \\ 2 \\ 2 Department of Biological Sciences, Marine Environmental Biology Section, University of Southern California, Los Angeles, CA, United States \\ 3 Center for Dark Energy Biosphere Investigations, University of Southern California, Los Angeles, California, USA \\ ${ }^{4}$ Chemical and Biological Signature Science Group, Pacific Northwest National Laboratory, Richland, Washington, USA \\ Corresponding Author: William C Nelson \\ Email address: william.nelson@pnnl.gov
}

Background: Advances in sequencing, assembly, and assortment of contigs into species-specific bins has enabled the reconstruction of genomes from metagenomic data (MAGs). Though a powerful technique, it is difficult to determine whether assembly and binning techniques are accurate when applied to environmental metagenomes due to a lack of complete reference genome sequences against which to check the resulting MAGs.

Methods: We compared MAGs derived from an enrichment culture containing $\sim 20$ organisms to complete genome sequences of 10 organisms isolated from the enrichment culture. Factors commonly considered in binning software - nucleotide composition and sequence repetitiveness - were calculated for both the correctly binned and not-binned regions. This direct comparison revealed biases in sequence characteristics and gene content in the not-binned regions. Additionally, the composition of three public data sets representing MAGs reconstructed from the Tara Oceans metagenomic data was compared to a set of representative genomes available through NCBI RefSeq to verify that the biases identified were observable in more complex data sets and using three contemporary binning software packages.

Results: Repeat sequences were frequently not binned in the genome reconstruction processes, as were sequence regions with variant nucleotide composition. Genes encoded on the not-binned regions were strongly biased towards ribosomal RNAs, transfer RNAs, mobile element functions and genes of unknown function. Our results support genome reconstruction as a robust process and suggest that reconstructions determined to be $>90 \%$ complete are likely to effectively represent organismal function, however, population-level genotypic heterogeneity in natural populations, such as uneven distribution of plasmids, can lead to incorrect inferences. 


\section{Biases in Genome Reconstruction from Metagenomic Data}

2

3 William C. Nelson ${ }^{1}$, Benjamin J. Tully ${ }^{2,3}$ and Jennifer M. Mobberley ${ }^{4}$

4

$5 \quad{ }^{1}$ Biological Sciences Division, Pacific Northwest National Laboratory, Richland, WA, USA

$6 \quad 2$ Department of Biological Sciences, Marine and Environmental Biology Section, University of

7 Southern California, Los Angeles, CA, USA

$8{ }^{3}$ Center for Dark Energy Biosphere Investigations, University of Southern California, Los

9 Angeles, CA, USA

$10{ }^{4}$ Chemical and Biological Signature Science Group, Pacific Northwest National Laboratory,

11 Richland, WA, USA

12

13 Corresponding Author:

14 William C. Nelson ${ }^{1}$

15 Pacific Northwest National Laboratory, PO Box 999, MSIN J4-18, Richland, WA 99352

16 william.nelson@pnnl.gov 


\section{ABSTRACT}

Background: Advances in sequencing, assembly, and assortment of contigs into species-specific bins has enabled the reconstruction of genomes from metagenomic data (MAGs). Though a powerful technique, it is difficult to determine whether assembly and binning techniques are accurate when applied to environmental metagenomes due to a lack of complete reference genome sequences against which to check the resulting MAGs.

Methods: We compared MAGs derived from an enrichment culture containing $\sim 20$ organisms to complete genome sequences of 10 organisms isolated from the enrichment culture. Factors commonly considered in binning software - nucleotide composition and sequence repetitiveness - were calculated for both the correctly binned and not-binned regions. This direct comparison revealed biases in sequence characteristics and gene content in the not-binned regions. Additionally, the composition of three public data sets representing MAGs reconstructed from the Tara Oceans metagenomic data was compared to a set of representative genomes available through NCBI RefSeq to verify that the biases identified were observable in more complex data sets and using three contemporary binning software packages.

Results: Repeat sequences were frequently not binned in the genome reconstruction processes, as were sequence regions with variant nucleotide composition. Genes encoded on the not-binned regions were strongly biased towards ribosomal RNAs, transfer RNAs, mobile element functions and genes of unknown function. Our results support genome reconstruction as a robust process and suggest that reconstructions determined to be $>90 \%$ complete are likely to effectively represent organismal function, however, population-level genotypic heterogeneity in natural populations, such as uneven distribution of plasmids, can lead to incorrect inferences. 


\section{INTRODUCTION}

High-throughput sequencing has revolutionized microbiology by circumventing "the great plate count anomaly" (1) and allowing direct investigation of natural communities in a cultureindependent manner (2-8). One goal of metagenomics has always been to obtain organismspecific, complete, genomic information from the complex mixture of sequence data generated from environmental samples. Having a complete genome sequence provides a platform for understanding the range of metabolic roles an organism can play within a community and the interactions it has with other organisms (9-11), and it can provide specific context for interpretation of transcriptomics and proteomics (12,13). Metagenome-assembled genomes (MAGs) are produced by segregating assembled contigs/scaffolds into organism-specific 'bins'. This process of genome reconstruction has benefitted from continuing advances in sequencing technologies, sequence assembly algorithms, and segregation methods (14). Early success assembling genomes from a simple community (15) has led to more recent studies reconstructing many organisms from complex environments (16-30). The accuracy of these techniques in the context of a complex environmental community is difficult to gauge, however, because most available complete microbial genome sequences that could serve as references are from cultured isolates, and these isolates are rarely present in environmental metagenomes. Techniques that have been developed to evaluate the accuracy of the binning process rely on conserved genes and consistency of nucleotide composition (31-35). These techniques, however, cannot make accurate determinations of how much sequence is missing or the functional potential of missing content. Genome reconstruction techniques have been tested using synthetic communities of cultured organisms (36) and simulated metagenomic datasets. Over time, increasingly sophisticated methods have been developed to simulate metagenomic read data sets, from the 
62 earlier Grinder (37), MetaSim (38), GemSIM (39), BEAR (40), and NeSSM (41), to the more

recent CAMISIM (42), which was developed as part of the community effort to address standards in metagenome analysis software development (43). Generally these simulators concern themselves with modeling community structure and sequencing attributes, such as read length and error rates, but are limited to presenting data generated from a reference genomic database, thus cannot model the genetic diversity found in most environments, although CAMISIM addresses this issue by implementing the genome evolution simulator sgEvolver (44). Because genetic variability within natural populations is, as yet, ill-defined (45), it is unlikely that such test data can accurately replicate the type and amount of variability found in natural communities, and the complications this variability causes.

Unicyanobacterial consortia (UCC) were developed as model systems to investigate the mechanisms of metabolic interaction between cyanobacteria and heterotrophs. These systems provide an opportunity to compare MAGs against a matching reference genome set and learn about potential gaps and pitfalls of current reconstruction processes. Two consortia, each containing a single unique cyanobacterial species and sharing an additional 18 heterotrophic species, were derived from a natural mat community (46). The communities have been sequenced, and genome reconstruction has been performed (47), yielding near-complete genome sequences revealing the presence and maintenance of microdiversity, such as might be found within an intact environmental sample. Thus, this system more accurately reflects in situ community diversity compared to synthetic communities constructed from isolated organisms. In parallel, isolates of 10 of the member species have also been sequenced $(47,48)$. This paired genomic and metagenomic data set allows direct comparison of MAGs from diverse organisms against 'ground truth' genomic data. Previously, we have shown that common aspects of the 
85

86

87

88

89

90

91

92

93

94

95

96

97

98

99

100

101

102

103

104

105

106

107

genome reconstruction process (assembly from a complex sequence space and segregation of contigs based on read depth profiles and sequence composition) to be both specific and sensitive (47).

We have investigated the nature of genomic regions that under current standard genome reconstruction techniques are not recovered (herein referred to as not-binned regions, or NRs) to evaluate how these regions differ from recovered regions (correctly binned regions, or CRs), and to what extent the missing genomic information might impact conclusions drawn from analysis of MAGs. Two common elements of current sequence segregation protocols are analysis of sequence composition and comparison of coverage profiles between samples, so we compared the nucleotide content of NRs vs CRs, examining both $\% \mathrm{G}+\mathrm{C}$ and tetranucleotide content, and the redundancy of sequence information both within the individual genome (i.e., repetitiveness within the genome) and across the entire metagenomic data set (i.e., sequence shared between populations). To determine the impact on downstream functional analyses, the gene content was examined for biases in the cellular roles of genes found within NRs and CRs.

To verify that the biases observed extended to more complex metagenomic datasets and across binning algorithms, the Tara Oceans metagenome, which has been binned by different groups using MetaBAT $(22,49)$, Anvi’o (31,50), and BinSanity $(21,51)$, was subjected to similar sequence and repeat compositional analysis.

\section{MATERIALS \& METHODS}

Data and Code Availability.

The UCC MAG and genome data analyzed are available in the GenBank repository as listed in Table 1. The metagenomic data used to construct the UCC MAGs is available from the NCBI 
108 SRA (accessions SRX1063989 and SRX1065184). MAGs reconstructed from the Tara Oceans

109 metagenomic data $(21,22)$ are available in the GenBank repository. MAGs from Delmont et al.

110 (50) are available through figshare (doi: 10.6084/m9.figshare.4902923). A list of MAGs and

111 corresponding identifiers are available in Supplemental Table 1. Complete bacterial and archaeal

112 genomes were collected from NCBI RefSeq (52) (accessed Aug 2019) based on assignment as

113 either "reference genome" or "representative genome" for the data column "refseq_category"

114 and "Complete Genome" in the "assembly_level" column. A list of genomes used in the analysis

115 are available in Supplemental Table 2. All analysis scripts are available at

116 http://github.com/wichne/biases_in_genome_reconstruction.

117 Identification of $C R$ and $N R$ regions.

118 The UCC scaffolds comprising each MAG were searched against their cognate complete genome

119 sequence using nucmer using the maxmatch option (53). Regions of the genomes that aligned

120 end-to-end to MAG scaffolds at $\geq 99 \%$ identity were cataloged as CR regions. All other genome

121 regions were considered NR regions.

122 Compositional analysis.

123 For the UCC MAGs and genomes, $\% \mathrm{G}+\mathrm{C}$ calculation and tetranucleotide frequency (TNF) chi-

124 square test were performed using custom Perl scripts (available at

125 http://github.com/wichne/biases_in_genome_reconstruction). Compositional analysis was

126 restricted to CR or NR regions longer than $1000 \mathrm{bp}$ to ensure sufficient sequence for meaningful

127 results. For TNF, the chi-squared statistic was calculated for each region using the TNF for the

128 whole genome as the expected values, and the mean and standard deviation for the CR and NR

129 pools calculated. For $\% \mathrm{G}+\mathrm{C}$ analysis, the mean $\% \mathrm{G}+\mathrm{C}$ for the $\mathrm{CR}$ and $\mathrm{NR}$ regions was

130 calculated, and the absolute difference was calculated between each region and the genome 
131 average, and average differences determined for CR and NR pools. To estimate $p$-values for the $132 \% \mathrm{G}+\mathrm{C}$ and $\mathrm{TNF}$ analyses, one thousand random coordinate sets yielding the same number and

133 length of fragments as in each genome's CR or NR set were generated from the genome

134 sequence and evaluated.

135 For comparison of the UCC data set to the Tara Oceans MAGs and RefSeq genome data 136 sets, sequence composition variance (i.e., deviation from the mean) was calculated for the $\% \mathrm{G}+\mathrm{C}$ 137 and tetranucleotide frequency using a custom Python script. The $\% \mathrm{G}+\mathrm{C}$ was calculated for $2 \mathrm{~kb}$ 138 segments (sliding window of 500bp) for each MAG or genome. A genome-wide variance value 139 was calculated for each MAG or genome based on the segments and plotted as a box plot per source data set. TNF was calculated for $10 \mathrm{~kb}$ segments (sliding window $5 \mathrm{~kb}$ ) for each MAG or

141 genome. Using the calculation described in Teeling (54), each segment had a Z-score calculated

142 for each tetranucleotide based on the observed-vs-expected frequency of the tetranucleotide in 143 the $10 \mathrm{~kb}$ segment. A Pearson correlation was then calculated in a pairwise fashion for all 144 segments. Variance of the Pearson correlation values within a MAG or genome was calculated 145 and plotted as a box plot per source data set.

\section{Repetitiveness analysis}

147 To calculate intragenome sequence repetitiveness, we determined the fraction of each genome 148 that was comprised of repeat sequence. Each genome sequence was searched against itself using 149 nucmer v3.0 (53) with the maxmatch option, and the lengths of regions that aligned to another 150 part of the genome/MAG with $\geq 97 \%$ identity were summed and divided by the length of the 151 genome/MAG.

152 To determine the repetitiveness of sequences across the entire metagenomic data set, 153 metagenome reads were searched against genome sequences using Bowtie2 (55). Per-base 
154 coverage was calculated using the samtools (56) depth command, and average coverage values

155 for the genomes, NRs and CRs were determined. One thousand sets of random coordinate

156 regions of the same number and lengths as in each set were analyzed to estimate p-values.

157 Results are reported as average coverage depth of NRs and CRs and the average difference from

158 the genome depth-of-coverage.

159 Gene function analysis

160 UCC complete genome sequences were annotated by the IMG pipeline (57), which included

161 COG assignment based on the December 2014 release of the 2003-2014 COGs (58). COGs

162 assigned to more than one functional category were counted for each assigned category. Genes

163 not assigned to a COG category were classified as 'unassigned'. Ribosomal RNA (rRNA) gene

164 features were identified by the IMG pipeline (59); transfer RNAs (tRNA) were identified with

165 tRNAscan-SE (60); other non-coding RNAs (ncRNA) were identified using the Rfam database

$166 \mathrm{v} 11.0$ (61) and infeRNAl v1.1 software (62). For each gene set, the category counts were

167 normalized to the total feature counts. Principle component analysis was performed and biplot of

168 gene categories was generated using R package bpca v.1.2-2 (http://cran.r-

169 project.org/web/packages/bpca/).

170 Statistical analysis.

171 Statistical tests were performed using modules within the Python package SciPy (63). The

172 normality of the calculated variance distributions for each set of genomes was determined using

173 the Shapiro-Wilk test (64). Genome sets with a normal distribution were compared to each other

174 with the T-test for two independent variables (65). Genome sets without a normal distribution

175 were compared to each other with the Mann-Whitney U test (66). p-values were adjusted for 
176 multiple comparisons with the Benjamini-Hochberg procedure (67) correction with a false

177 discovery rate of 25\% (Supplemental Table 3).

178

179 RESULTS AND DISCUSSION

180 The power of metagenomics is that it allows exploration of diverse communities from which we

181 cannot culture the component populations either because the proper growth conditions are

182 unknown or difficult to replicate in a laboratory environment, or simply because there are too

183 many organisms present to have the resources or time to pursue the effort. Because of this, there

184 are very few examples of sequenced organisms isolated from the same sample from which

185 metagenomic sequencing and binning has been done to generate MAGs. As such, a 'gold

186 standard' for evaluation of MAG content has been difficult to come by. We have taken

187 advantage of two enrichment cultures from which MAGs and isolate genomes have been derived

188 to generate just such a 'gold standard' comparison framework. We have previously generated

189 two unicyanobacterial consortial cultures (UCC) - enrichment cultures each containing a distinct

190 cyanobacterial population and different, yet overlapping, communities of associated

191 heterotrophs, each numbering $<20$ species - and performed metagenomic sequencing, assembly

192 and binning.(47,48). Illumina $150 \mathrm{bp}$ paired-end reads were generated from each community,

193 and IDBA_ud was used to assemble the read sets separately and in co-assembly. The abundances

194 of the organisms differed between the two communities, allowing us to bin the sequences by

195 comparing sequence coverage values of contigs between the two UCCs in a predominantly

196 manual process (inspired by the work of Dick, et al (68)). The resulting MAGs were manually

197 curated to eliminate contaminating contigs and identify mis-binned contigs, correctly placing

198 them when possible. In parallel, ten organisms were isolated from the UCCs and completely 
199 sequenced. Comparison of the MAGs to the isolate genomes showed recovery of $>90 \%$ of

200 sequence for genomes with at least 10x coverage, with one exception, Halomonas sp. HL-93,

201 which had 85\% recovery from $11 x$ coverage (Table 1). Co-linear sequence alignments indicated

202 there were no assembly errors in the binned contigs (47, and data not shown). Based on the

203 isolate-MAG comparisons, NRs were identified. Porphyrobacter HL-46 had the lowest

204 metagenome coverage (3.6x). Its MAG comprised hundreds of short contigs and was determined

205 to be $\sim 40 \%$ complete. Thus, the NRs for HL-46 are assumed to be primarily caused by the

206 random sampling of the shotgun sequencing methodology and not by any inherent content

207 biases, allowing the HL-46 analyses to serve as a control.

208 To determine if NRs were not binned due to lack of assembly, we mapped the contigs from the

209 assembly to the CR and NR regions of the genomes and looked at the contig coverage of the

210 regions. As expected, the CRs showed an average contig coverage of $1.04 \pm 0.14$, and most

211 regions had only a single contig map to them (Fig S1). Many of the cases of multiple contigs

212 mapping to a CR were due to short ( $<200 \mathrm{bp})$ contigs of repeat sequence which might be an

213 artifact of the assembler (IDBA_ud). NRs show a strong positive correlation between region

214 length and number of contigs mapping, with an average coverage of $0.94 \pm 0.71$ (Fig S2). This

215 suggests poorer assembly of the NRs and higher repeat content, but also indicates that most NR

216 sequence is present in the contig set, and thus the binning process is the main determinant of

217 NRs.

218

219 Nucleotide composition of NRs frequently differs from the genome average

220 Bacteria and Archaea have evolved to have a fairly consistent $\% \mathrm{G}+\mathrm{C}$ across their genome (69),

221 so much so that it has been proposed as a metric of classification at higher taxonomic levels (70). 
222 It is not uncommon, however, to observe regions within a genome that differ significantly from

223 the genome average (71). This variation can be the result of selective pressure for structural

224 properties in non-coding genes, for instance ribosomal RNAs and other functional RNAs have

225 been shown to vary in nucleotide composition in correlation with optimal growth temperature

226 (72). In other cases, divergent $\% \mathrm{G}+\mathrm{C}$ indicates a region which has been acquired recently (in

227 evolutionary time) from a non-related source (i.e., horizontal gene transfer) (73). To investigate

228 whether variant $\mathrm{G}+\mathrm{C}$ confounds genome reconstruction, we compared the $\% \mathrm{G}+\mathrm{C}$ of $\mathrm{NRs}$ to that

229 of CRs and the complete genome.

230 The genomes in this study had a range of $\% \mathrm{G}+\mathrm{C}$ values, from $42 \%$ (A. marincola $\mathrm{HL}-49)$

231 to $68 \%$ (Erythrobacteraceae bacterium HL-111), with most skewing toward the higher values

232 (Table 2). We determined the $\% \mathrm{G}+\mathrm{C}$ for each $\mathrm{CR}$ and $\mathrm{NR} \geq 200 \mathrm{bp}$ in length and compared them

233 to the $\% \mathrm{G}+\mathrm{C}$ for the complete genome. For genomes with more than one genomic element, each

234 molecule was considered separately since extrachromosomal elements may have distinct

235 nucleotide composition. For seven of the genomes, the $\% \mathrm{G}+\mathrm{C}$ for the $\mathrm{NRs}$ differed significantly

$236(\mathrm{p} \leq 0.005)$ from the genome average, while the CRs generally reflected the genome average

237 (Table 2). The $\% \mathrm{G}+\mathrm{C}$ averages for NRs from HL-48 and HL-111 were significantly lower

238 (45.76\% and $64.26 \%$, respectively) than the genomes' averages $(58.98 \%$ and $68.12 \%$

239 respectively). Other genomes (HL-53, HL-55, HL-109) had some NRs with \%G+C higher than

240 the genome average and some NRs with lower values (Figure 1), despite having different

241 average $\% \mathrm{G}+\mathrm{C}$ values $(47.5 \%, 56.0 \%$ and $64.1 \%$ respectively). Extrachromosomal elements

242 analyzed did not display a significant difference in the $\% \mathrm{G}+\mathrm{C}$ of their $\mathrm{NRs}$ from the molecule

243 average. As expected, the values for the NRs and CRs of HL-46 showed no significant difference

244 from the genome average (Table 2), however, HL-46's CRs and NRs did not display identical 
$245 \% \mathrm{G}+\mathrm{C}$ profiles (Figure 1). There was a slight bias toward higher $\% \mathrm{G}+\mathrm{C}$ for the $\mathrm{NRs}$ and lower

$246 \% \mathrm{G}+\mathrm{C}$ in the $\mathrm{CRs}$, which could reflect a bias in the assembly algorithm.

247 Tetranucleotide frequency (TNF) has been shown to be capable of distinguishing higher up to the species level

248 taxonomic classifications, up to species $(54,68)$. This resolving power has been leveraged in

249 binning protocols $(15,74-76)$. To investigate whether genomic regions with divergent TNF are

250 poorly recovered in genome reconstruction, we compared the TNFs of CRs and NRs to that of

251 the cognate complete genome using chi-squared analysis. In most cases, the chi-squared statistic

252 was an order of magnitude higher for NRs versus CRs, and the differences were significant for

253 all chromosomal sequences except for HL-46, HL-109, HL-93 and the small chromosome of

254 HL-91 (Table 3).

255 One factor that could affect nucleotide composition effects on binning is the length of the 256 region with divergent composition versus the length of the contig. If the variant region comprises

257 most of the length of the contig being evaluated, the difference from the genome average will be 258 pronounced, whereas if the divergent region is only a small percentage of the contig length, the signal will be muted. An examination of CR/NR length versus compositional variance (Fig. S3) revealed a strong, significant negative correlation between contig length and TNF chi square for

12). Taken together, the $\% \mathrm{G}+\mathrm{C}$ and $\mathrm{TNF}$ results show that genomic regions with divergent

for short contigs. The most effective way to overcome this problem is to enhance assembly such that regions with unusual content are included in significantly longer contigs, or, through clone linkage, identify strong, unique connections to binned contigs. 


\section{Repeated sequences segregate aberrantly}

269 Sequence coverage profiles are frequently effective in discriminating contigs from different organisms (15). Samples taken under different conditions or at different times capture

271 community states which have similar organismal composition but differing relative abundances.

272 This difference translates to distinct coverage profiles for assembled contigs, and thus contigs

273 with similar coverage profiles are assumed to originate from the same organism. In this data set,

274 for example, we compared two cultures with near-identical heterotroph species composition, but different cyanobacteria acting as a conduit for energy and carbon $(46,47)$. Other studies have compared samples taken at different times (75). Coverage analysis is more difficult for repeated regions of a genome, which will yield higher coverage values than the genome average and thus are more likely to be either not binned or binned improperly. Differential coverage analysis can mitigate this problem by identifying correlated changes in abundance of contigs with different coverage. Unlike nucleotide composition variance, however, unusual high-coverage signal due to repeat sequence is less likely to be diluted by incorporation into a larger contig because assemblers (especially standard de Bruijn graph assemblers using short-read data) tend to terminate contigs when repeats are encountered and/or assemble repeats into separate contigs (77).

To examine the impact of repeated sequences on genome reconstruction, we determined the repetitiveness of sequence information across CRs and NRs, determined from a self-versusself similarity search, and compared those values to the genome average. Correspondence of repeated regions and NRs was strong (Figures 2 and 3, Figure S4). In HL-111, all NRs save one repeat content equal to or lower than the genome average. 
Another phenomenon that can affect contig coverage in metagenomic assembly is

292

293

294

295

296

297

multiple organisms sharing identical regions of DNA. Some regions are highly conserved between related species, an example being the ribosomal RNA operon, which is known to confound assemblers and segregation strategies (78). Alternatively, mobile elements such as plasmids or transposons can have a broad host range and invade and inhabit closely or even distantly related organisms (79). Such regions, even if not repeated within a genome, will exhibit anomalous coverage and thus could be either excluded or mis-binned. We examined the metagenomic read coverage depth to determine if NRs had anomalous profiles relative to the whole genome and the CRs. For most reconstructions, the NRs' coverage differed from the genome average and that of the CRs (Table 4, Fig 2, Fig S4). Only HL-46 and one of the HL109 molecules did not have significant differences. Most NRs displayed higher or equivalent coverage values, however, several NRs in HL-48 and the two small plasmids associated with HL-91 showed lower metagenomic coverage values (Figure S4). A likely explanation for this is the presence in the consortia of sub-populations of these organisms that lack the plasmids.

\section{Functional assessment of NR genes}

To determine the extent to which regions missing from reconstructions might affect downstream metabolic or functional analyses and predictions for organisms and communities, we examined the gene content of the NRs and the functional roles of those genes. COG categorization was used as a basis for comparison because of its ability to identify, in particular, genes associated with mobile elements such as plasmids, phage and insertion sequences. In addition, we evaluated the distribution of non-coding RNA genes since some are known to be repeated within genomes 
313 (multiple rRNA operons, for example), and others (tRNAs) are commonly associated with 314 mobile elements (80).

315 For all the reconstructions, the gene content of the NRs differed from that of the CRs and

316 complete genomes. Functional analysis of gene sequences shows that this difference was largely

317 driven by genes encoding mobile element functions (COG category X) and RNA genes (Figure

318 4). The mobile element genes in the NR regions were predominantly transposases with some

319 contribution from bacteriophage and plasmid genes (HL-91; HL-93). Most of the identified

rRNA genes fell within NRs, with only HL-48 and HL-53 each having one rRNA contained in a

321 CR. In addition, the NRs, including the two entire plasmids from HL-91 which were not binned, contained a higher percentage of genes that were not assigned to a COG category.

323

Evaluation of a complex metagenomic data set and common automated binning tools

325

326

327

328

329

330

331

332

333

334

335

To verify that our conclusions of genome reconstruction bias in the highly curated UCC data set were extendable to more complex data sets and for alternate, widely-used binning tools, we applied similar analyses to MAGs generated from the Tara Oceans metagenomic data using distinct genome reconstruction protocols. For this comparison, 4,557 MAGs generated from the Tara Oceans microbial metagenomic data reconstructed using three complementary methods were collected and analyzed. Three different automated binning methodologies were employed to generate the MAG data set: MetaBat (v0.26.3) $(22,49)$, BinSanity (v1.0) $(21,51)$, and CONCOCT (with manual refinement in anvi'o) (31,50). All three automated binning algorithms utilized read coverage and TNF to identify congruent contigs, with the intended role of the algorithms to reconstruct high confidence environmental genomes while avoiding over-binning (i.e., removing elements that deviate from the mean values of the binned contigs). The MAGs 
336 had a mean estimated completeness and contamination of $76.6 \%$ and $2.2 \%$, respectively, as

337 determined by CheckM v.1.1.1 (32). In comparison, 1,736 'representative' and 'reference'

338 complete genomes were collected from NCBI RefSeq.

339 Our results above predicted that the MAGs would have lower $\% \mathrm{G}+\mathrm{C}$ variance and TNF

340 variance than the isolate complete genome data set. For the observed $\% \mathrm{G}+\mathrm{C}$, MAGs tended to

341 have lower variance $(p<0.001)$ than isolate genomes (Figure 5A). The exception was the Parks

342 et al. MAGs, which had a much larger variance, even compared to the RefSeq genome set (mean

343 vs mean, $p<0.001)$. This may be the result of the additional step applied to the MAGs by Parks

344 et al., whereby related MAGs with $<3 \%$ mean $\% \mathrm{G}+\mathrm{C}$ difference were merged into a single

345 representative MAG (22). For the Tully et al. and Delmont et al. MAGs, the lower variance

346 observed compared to the RefSeq genomes is likely due to removal of contigs with deviant

$347 \% \mathrm{G}+\mathrm{C}$ values during binning $(21,50)$. The MAGs also had lower variance with regards to TNF

348 compared to the RefSeq genomes $(p<0.001)$ (Figure 5B), again, likely due to genomic elements

349 that deviated from the average value of the binned contigs having been removed during the

350 binning steps. These observations support our conclusions regarding genome regions having

351 divergent nucleotide composition being underrepresented in MAGs.

The Tara and NCBI Refseq data sets were then evaluated for repeat sequence content.

353 Each MAG and isolate genome was compared to itself using NUCmer to identify the fraction of

354 the genome composed of repeat regions (regions with $\geq 97 \%$ sequence identity). MAGs

355 universally had a smaller fraction of genomic information in repeat regions compared to isolate

356 genomes $(p<0.01$; Figure 6). The lack of repeat regions in MAGs is likely the result of repeated

357 regions having inflated or depressed read coverage values relative to the mean of the genome,

358 depending on the number of copies of the repeat region present in the genome and how stable 
359 this number is across the population. Compared to the other Tara MAGs, the Tully et al. MAGs

360 had a larger fraction of redundant genomic elements. It is unclear what aspect of the assembly

361 and binning methodology has influenced these results. On average, the lengths of the repeat

362 regions from the Tully et al. MAGs are longer than the repeat regions in the RefSeq genomes

363 (mean: 1,052bp vs 868bp, respectively).

364

What's missing from reconstructed genomes?

366

367

368

369

370

371

372

373

374

375

376

377

378

379

380

381

Analysis of regions that were not recovered from genome reconstruction (NRs) showed both nucleotide compositional variance and intragenome repetitiveness. The $\% \mathrm{G}+\mathrm{C}$ and tetranucleotide frequencies of NRs tended to differ from that of complete genomes (Tables 2 and 3, Figure 1), and the sequence coverage differed. This met expectations since, in general, binning tools are designed around the assumption that sequences with similar properties belong together, thus any genome region that varies significantly from the genome average is likely be incorrectly binned if it comprises the majority of a contig under consideration. Regions with atypical nucleotide content have been observed to contain genes upon which selective pressures are acting on nucleic acid structure, such as ribosomal RNAs and tRNAs $(72,81,82)$, and exogenously introduced segments such as mobile elements $(83,84)$. It is significant that many of the NRs displayed lower $\% \mathrm{G}+\mathrm{C}$ than the genome average, since it has been observed that laterally acquired regions tend to have lower $\% \mathrm{G}+\mathrm{C}$ than their hosts $(83)$, as phage and insertion sequences tend to have $\mathrm{A}+\mathrm{T}$-enriched genomes (85). Notably, many genome regions with variant nucleotide composition were incorporated into longer contigs by the assembler, masking the variance and allowing correct binning. Conversely, the assembler collapsed repeated region sequences into single contigs, and thus they were not binned due to the inflated sequence 
382 coverage values. Often, repeated sequences displayed divergent nucleotide composition, but the

383 reciprocal was less frequent, indicating that repetitiveness is the stronger driver of binning

384 failure. These results demonstrate that assembly efficiency is an important determining factor for

385 correct binning, or conversely, any factor that results in shorter assemblies will result in poorer

386 recovery of anomalous regions. Thus, it is advisable to include replication and positive controls

387 in metagenomic sequencing protocols, particularly for highly diverse communities such as soils

388 and riverbed sediments, to allow evaluation of assembly efficiency and accuracy.

389 Repeat regions identified in this study appeared to largely consist of insertion elements

390 based on functional analysis and their relatively short size (1-2 kb). Failure of these regions to be

391 correctly binned is unlikely to meaningfully affect functional predictions for a reconstructed

392 genome. Their presence in a genome is more likely to affect metabolic reconstruction analysis by

393 reducing assembly efficiency, resulting in more, shorter contigs and increasing the chance that

394 these shorter contigs are not binned or incorrectly binned. Technological advances increasing

395 read length beyond $2 \mathrm{~kb}$ will increase contig lengths, binning accuracy, and the likelihood of

396 yielding closed genomes from environmental samples $(8,86,87)$.

397 NRs were generally observed to be short, with a median length of less than $5 \mathrm{~kb}$ (Table

398 1) and containing only a handful of genes. Thus, even a MAG with many gaps (indicating a large

399 number of NRs) may be missing only a small percentage of its genome. The conserved single-

400 copy gene (CSCG) estimations for completeness appear for all intents and purposes to be a

401 reasonable indication of how much information is absent (47). One caveat to this conclusion,

402 however, is that extrachromosomal elements, plasmids and phages (integrated or otherwise)

403 typically do not carry CSCG markers, and thus are essentially invisible in such analyses. The

404 longer NRs observed in our analysis appear to comprise integrated plasmids or phage, and thus 
405

406

407

408

409

410

411

412

413

414

415

416

417

418

419

420

421

422

423

424

425

426

427

any gap in a reconstruction could represent up to $50 \mathrm{~kb}$ (or more) of genetic material.

Importantly, these represent introduced genetic material, which, while likely conveying a beneficial trait, are unlikely to carry functions that are integral to host metabolic function.

\section{CONCLUSIONS}

This analysis indicates that reconstructed genomes estimated to be near-complete can be assumed to contain nearly all genes important to metabolic reconstruction. The majority of identifiable genes present on NRs appear to be either highly conserved, non-coding genes that can be assumed to be present (such as the rRNA genes and tRNA genes) or are associated with mobile genetic elements. While many of these genes may be not be directly related to cellular metabolism (transposases, toxin/antitoxin systems, phage and plasmid functions), it should be noted that entire extrachromosomal elements may be missed by the binning process due to either alternate nucleotide composition, a higher number of copies per cell than the genome, or occupancy in only a subset of the population (such as the two molecules in HL-109). These elements frequently carry genes that alter the physiology or resistance of the host organism. For example, HL-109 and HL-111 have NRs that includes genes involved in glycan biosynthesis, suggesting alterations to the cell wall, while HL-91 has picked up a multidrug efflux transporter. As such, reconstructed genomes can be considered reliable foundations for metabolic reconstruction but should not be assumed to be comprehensive for the function of the organism.

\section{ACKNOWLEDGEMENTS}

The authors would like to thank Jim Fredrickson and Lori Nelson for critical evaluation of the manuscript during preparation. W.C.N. and J.M.M. were funded through the U.S. Department of 
Research (OBER), and this work was a contribution of the Pacific Northwest National

for Dark Energy Biosphere Investigations (OCE- 0939654). This is C-DEBI Contribution XXX.

\section{REFERENCES}

434

1. Staley JT, Konopka A. Measurement of in Situ Activities of Nonphotosynthetic Microorganisms in Aquatic and Terrestrial Habitats. Annu Rev Microbiol. 1985;39(1):321-46.

2. Venter JC, Remington K, Heidelberg JF, Halpern AL, Rusch D, Eisen JA, et al. Environmental genome shotgun sequencing of the Sargasso Sea. Science. 2004 Apr 2;304(5667):66-74.

3. DeLong EF, Preston CM, Mincer T, Rich V, Hallam SJ, Frigaard N-U, et al. Community Genomics Among Stratified Microbial Assemblages in the Ocean's Interior. Science. 2006 Jan 27;311(5760):496-503.

4. Costello EK, Lauber CL, Hamady M, Fierer N, Gordon JI, Knight R. Bacterial Community Variation in Human Body Habitats Across Space and Time. Science. 2009 Dec 18;326(5960):16947.

5. Caporaso JG, Lauber CL, Walters WA, Berg-Lyons D, Huntley J, Fierer N, et al. Ultra-highthroughput microbial community analysis on the Illumina HiSeq and MiSeq platforms. ISME J. 2012 Aug;6(8):1621-4.

6. Zhou J, He Z, Yang Y, Deng Y, Tringe SG, Alvarez-Cohen L. High-throughput metagenomic technologies for complex microbial community analysis: open and closed formats. MBio. 2015 Jan $27 ; 6(1)$.

7. Rinke C, Schwientek P, Sczyrba A, Ivanova NN, Anderson IJ, Cheng J-F, et al. Insights into the phylogeny and coding potential of microbial dark matter. Nature. 2013 Jul;499(7459):431-7.

8. White RA, Bottos EM, Roy Chowdhury T, Zucker JD, Brislawn CJ, Nicora CD, et al. Moleculo Long-Read Sequencing Facilitates Assembly and Genomic Binning from Complex Soil Metagenomes. mSystems. 2016 Jun;1(3).

9. Iverson V, Morris RM, Frazar CD, Berthiaume CT, Morales RL, Armbrust EV. Untangling Genomes from Metagenomes: Revealing an Uncultured Class of Marine Euryarchaeota. Science. 2012 Feb 3;335(6068):587-90.

10. Sharon I, Morowitz MJ, Thomas BC, Costello EK, Relman DA, Banfield JF. Time series community genomics analysis reveals rapid shifts in bacterial species, strains, and phage during infant gut colonization. Genome Res. 2013 Jan 1;23(1):111-20. 
461

462

463

464

465

466

467

468

469

470

471

472

473

474

475

476

477

478

479

480

481

482

483

484

485

486

487

488

489

490

491

492

493

494

495

496

11. Delmont TO, Eren AM, Vineis JH, Post AF. Genome reconstructions indicate the partitioning of ecological functions inside a phytoplankton bloom in the Amundsen Sea, Antarctica. Front Microbiol [Internet]. 2015 [cited $2020 \mathrm{Jul}$ 5];6. Available from: https://www.frontiersin.org/articles/10.3389/fmicb.2015.01090/full

12. Lesniewski RA, Jain S, Anantharaman K, Schloss PD, Dick GJ. The metatranscriptome of a deepsea hydrothermal plume is dominated by water column methanotrophs and lithotrophs. ISME J. 2012 Dec;6(12):2257-68.

13. Ram RJ, VerBerkmoes NC, Thelen MP, Tyson GW, Baker BJ, Blake RC, et al. Community Proteomics of a Natural Microbial Biofilm. Science. 2005 Jun 24;308(5730):1915-20.

14. Sangwan N, Xia F, Gilbert JA. Recovering complete and draft population genomes from metagenome datasets. Microbiome. 2016 Mar 8;4:8.

15. Tyson GW, Chapman J, Hugenholtz P, Allen EE, Ram RJ, Richardson PM, et al. Community structure and metabolism through reconstruction of microbial genomes from the environment. Nature. 2004 Mar 4;428(6978):37-43.

16. Brown CT, Hug LA, Thomas BC, Sharon I, Castelle CJ, Singh A, et al. Unusual biology across a group comprising more than 15\% of domain Bacteria. Nature. 2015 Jul 9;523(7559):208-U173.

17. Anantharaman K, Breier JA, Dick GJ. Metagenomic resolution of microbial functions in deep-sea hydrothermal plumes across the Eastern Lau Spreading Center. ISME J. 2016 Jan;10(1):225-39.

18. Baker BJ, Lazar CS, Teske AP, Dick GJ. Genomic resolution of linkages in carbon, nitrogen, and sulfur cycling among widespread estuary sediment bacteria. Microbiome. 2015;3:14.

19. Li M, Baker BJ, Anantharaman K, Jain S, Breier JA, Dick GJ. Genomic and transcriptomic evidence for scavenging of diverse organic compounds by widespread deep-sea archaea. Nat Commun. 2015 Nov 17;6:8933.

20. Nobu MK, Narihiro T, Rinke C, Kamagata Y, Tringe SG, Woyke T, et al. Microbial dark matter ecogenomics reveals complex synergistic networks in a methanogenic bioreactor. ISME J. 2015 Aug;9(8):1710-22.

21. Tully BJ, Graham ED, Heidelberg JF. The reconstruction of 2,631 draft metagenome-assembled genomes from the global oceans. Sci Data. 2018 Jan 16;5:170203.

22. Parks DH, Rinke C, Chuvochina M, Chaumeil PA, Woodcroft B, Evans PN, et al. Recovery of nearly 8,000 metagenome-assembled genomes substantially expands the tree of life. Nat Microbiol. $2018 \mathrm{Feb} ; 3(2): 253-253$.

23. Pasolli E, Asnicar F, Manara S, Zolfo M, Karcher N, Armanini F, et al. Extensive Unexplored Human Microbiome Diversity Revealed by Over 150,000 Genomes from Metagenomes Spanning Age, Geography, and Lifestyle. Cell. 2019 Jan;176(3):649-662.e20.

24. Almeida A, Mitchell AL, Boland M, Forster SC, Gloor GB, Tarkowska A, et al. A new genomic blueprint of the human gut microbiota. Nature. 2019 Apr;568(7753):499-504. 
497

498

499

500

501

502

503

504

505

506

507

508

509

510

511

512

513

514

515

516

517

518

519

520

521

522

523

524

525

526

527

528

529

530

531

532

533
25. Mobberley JM, Lindemann SR, Bernstein HC, Moran JJ, Renslow RS, Babauta J, Hu D, Beyenal H, Nelson WC. Organismal and spatial partitioning of energy and macronutrient transformations within a hypersaline mat. FEMS Microbiol Ecol [Internet]. 2017 Apr 1 [cited 2020 Sep 2];93(4). Available from: https://academic.oup.com/femsec/article/doi/10.1093/femsec/fix028/3071443

26. Stewart RD, Auffret MD, Warr A, Walker AW, Roehe R, Watson M. Compendium of 4,941 rumen metagenome-assembled genomes for rumen microbiome biology and enzyme discovery. Nat Biotechnol. 2019;37(8):953-61.

27. Pedron R, Esposito A, Bianconi I, Pasolli E, Tett A, Asnicar F, Cristofolini M, Segata N, Jousson O. Genomic and metagenomic insights into the microbial community of a thermal spring. Microbiome. 2019 Dec;7(1):8.

28. Wong HL, White RA, Visscher PT, Charlesworth JC, Vázquez-Campos X, Burns BP. Disentangling the drivers of functional complexity at the metagenomic level in Shark Bay microbial mat microbiomes. ISME J. 2018 Nov;12(11):2619-39.

29. Daly RA, Borton MA, Wilkins MJ, Hoyt DW, Kountz DJ, Wolfe RA, Welch SA, Marcus DN, Trexler RV, MacRae JD, Krzycki JA, Cole DR, Mouser PJ, Wrighton KC. Microbial metabolisms in a 2.5-km-deep ecosystem created by hydraulic fracturing in shales. Nat Microbiol. 2016 Oct;1(10):16146.

30. Danczak RE, Johnston MD, Kenah C, Slattery M, Wrighton KC, Wilkins MJ. Members of the Candidate Phyla Radiation are functionally differentiated by carbon- and nitrogen-cycling capabilities. Microbiome. 2017 Dec;5(1):112.

31. Eren AM, Esen OC, Quince C, Vineis JH, Morrison HG, Sogin ML, et al. Anvi'o: an advanced analysis and visualization platform for 'omics data. PeerJ. 2015;3:e1319.

32. Parks DH, Imelfort M, Skennerton CT, Hugenholtz P, Tyson GW. CheckM: assessing the quality of microbial genomes recovered from isolates, single cells, and metagenomes. Genome Res. 2015 Jul;25(7):1043-55.

33. Waterhouse RM, Seppey M, Simão FA, Manni M, Ioannidis P, Klioutchnikov G, et al. BUSCO Applications from Quality Assessments to Gene Prediction and Phylogenomics. Mol Biol Evol. 2018 Mar 1;35(3):543-8.

34. Chen L-X, Anantharaman K, Shaiber A, Eren AM, Banfield JF. Accurate and Complete Genomes from Metagenomes. bioRxiv. 2019 Oct 29;808410.

35. Hugoson E, Lam WT, Guy L. miComplete: weighted quality evaluation of assembled microbial genomes. Hancock J, editor. Bioinformatics. 2019 Aug 22;btz664.

36. Hardwick SA, Chen WY, Wong T, Kanakamedala BS, Deveson IW, Ongley SE, et al. Synthetic microbe communities provide internal reference standards for metagenome sequencing and analysis. Nat Commun. 2018 Dec;9(1):3096.

37. Angly FE, Willner D, Rohwer F, Hugenholtz P, Tyson GW. Grinder: a versatile amplicon and shotgun sequence simulator. Nucleic Acids Res. 2012 Jul 1;40(12):e94-e94. 
534

535

536

537

538

539

540

541

542

543

544

545

546

547

548

549

550

551

552

553

554

555

556

557

558

559

560

561

562

563

564

565

566

567

568

38. Richter DC, Ott F, Auch AF, Schmid R, Huson DH. MetaSim-A Sequencing Simulator for Genomics and Metagenomics. Field D, editor. PLoS ONE. 2008 Oct 8;3(10):e3373.

39. McElroy KE, Luciani F, Thomas T. GemSIM: general, error-model based simulator of nextgeneration sequencing data. BMC Genomics. 2012;13(1):74.

40. Johnson S, Trost B, Long JR, Pittet V, Kusalik A. A better sequence-read simulator program for metagenomics. BMC Bioinformatics. 2014 Sep;15(S9):S14.

41. Jia B, Xuan L, Cai K, Hu Z, Ma L, Wei C. NeSSM: A Next-Generation Sequencing Simulator for Metagenomics. Janssen PJ, editor. PLoS ONE. 2013 Oct 4;8(10):e75448.

42. Fritz A, Hofmann P, Majda S, Dahms E, Dröge J, Fiedler J, et al. CAMISIM: simulating metagenomes and microbial communities. Microbiome. 2019 Dec;7(1):17.

43. Sczyrba A, Hofmann P, Belmann P, Koslicki D, Janssen S, Dröge J, et al. Critical Assessment of Metagenome Interpretation - a benchmark of metagenomics software. Nat Methods. 2017 Nov;14(11):1063-71.

44. Darling ACE. Mauve: Multiple Alignment of Conserved Genomic Sequence With Rearrangements. Genome Res. 2004 Jun 14;14(7):1394-403.

45. Rocha EPC. Neutral Theory, Microbial Practice: Challenges in Bacterial Population Genetics. Mol Biol Evol. 2018 Jun 1;35(6):1338-47.

46. Cole JK, Hutchison JR, Renslow RS, Kim YM, Chrisler WB, Engelmann HE, et al. Phototrophic biofilm assembly in microbial-mat-derived unicyanobacterial consortia: model systems for the study of autotroph-heterotroph interactions. Front Microbiol. 2014;5:109.

47. Nelson WC, Maezato Y, Wu YW, Romine MF, Lindemann SR. Identification and Resolution of Microdiversity through Metagenomic Sequencing of Parallel Consortia. Appl Env Microbiol. 2015;82(1):255-67.

48. Romine MF, Rodionov DA, Maezato Y, Osterman AL, Nelson WC. Underlying mechanisms for syntrophic metabolism of essential enzyme cofactors in microbial communities. ISME J. 2017;

49. Kang DD, Froula J, Egan R, Wang Z. MetaBAT, an efficient tool for accurately reconstructing single genomes from complex microbial communities. PeerJ. 2015;3:e1165.

50. Delmont TO, Quince C, Shaiber A, Esen ÖC, Lee ST, Rappé MS, et al. Nitrogen-fixing populations of Planctomycetes and Proteobacteria are abundant in surface ocean metagenomes. Nat Microbiol. 2018 Jul;3(7):804-13.

51. Graham ED, Heidelberg JF, Tully BJ. BinSanity: unsupervised clustering of environmental microbial assemblies using coverage and affinity propagation. PeerJ. 2017 Mar 8;5:e3035.

52. O'Leary NA, Wright MW, Brister JR, Ciufo S, Haddad D, McVeigh R, et al. Reference sequence (RefSeq) database at NCBI: current status, taxonomic expansion, and functional annotation. Nucleic Acids Res. 2016 Jan 4;44(D1):D733-745.

PeerJ reviewing PDF | (2017:03:17041:1:1:NEW 4 Sep 2020) 
569

570

571

572

573

574

575

576

577

578

579

580

581

582

583

584

585

586

587

588

589

590

591

592

593

594

595

596

597

598

599

600

601

602

603

53. Kurtz S, Phillippy A, Delcher AL, Smoot M, Shumway M, Antonescu C, et al. Versatile and open software for comparing large genomes. Genome Biol. 2004;5(2).

54. Teeling H, Meyerdierks A, Bauer M, Amann R, Glockner FO. Application of tetranucleotide frequencies for the assignment of genomic fragments. Env Microbiol. 2004 Sep;6(9):938-47.

55. Langmead B, Salzberg SL. Fast gapped-read alignment with Bowtie 2. Nat Methods. 2012 Apr;9(4):357-U54.

56. Li H, Handsaker B, Wysoker A, Fennell T, Ruan J, Homer N, et al. The Sequence Alignment/Map format and SAMtools. Bioinformatics. 2009 Aug 15;25(16):2078-9.

57. Huntemann M, Ivanova NN, Mavromatis K, Tripp HJ, Paez-Espino D, Palaniappan K, et al. The standard operating procedure of the DOE-JGI Microbial Genome Annotation Pipeline (MGAP v.4). Stand Genomic Sci. 2015 Oct 26;10.

58. Galperin MY, Makarova KS, Wolf YI, Koonin EV. Expanded microbial genome coverage and improved protein family annotation in the COG database. Nucleic Acids Res. 2015 Jan 28;43(D1):D261-9.

59. Markowitz VM, Chen IM, Palaniappan K, Chu K, Szeto E, Pillay M, et al. IMG 4 version of the integrated microbial genomes comparative analysis system. Nucleic Acids Res. 2014 Jan;42(Database issue):D560-7.

60. Lowe TM, Eddy SR. tRNAscan-SE: A program for improved detection of transfer RNA genes in genomic sequence. Nucleic Acids Res. 1997 Mar 1;25(5):955-64.

61. Burge SW, Daub J, Eberhardt R, Tate J, Barquist L, Nawrocki EP, et al. Rfam 11.0: 10 years of RNA families. Nucleic Acids Res. 2013 Jan;41(D1):D226-32.

62. Nawrocki EP, Eddy SR. Infernal 1.1: 100-fold faster RNA homology searches. Bioinformatics. 2013 Nov 15;29(22):2933-5.

63. Virtanen P, Gommers R, Oliphant TE, Haberland M, Reddy T, Cournapeau D, et al. SciPy 1.0-Fundamental Algorithms for Scientific Computing in Python. ArXiv190710121 Phys [Internet]. 2019 Jul 23 [cited 2020 Jan 2]; Available from: http://arxiv.org/abs/1907.10121

64. Shapiro SS, Wilk MB. An Analysis of Variance Test for Normality (Complete Samples). Biometrika. 1965 Dec;52(3/4):591.

65. Welch BL. THE GENERALIZATION OF 'STUDENT'S' PROBLEM WHEN SEVERAL DIFFERENT POPULATION VARLANCES ARE INVOLVED. Biometrika. 1947 Jan 1;34(12):28-35.

66. Mann HB, Whitney DR. On a Test of Whether one of Two Random Variables is Stochastically Larger than the Other. Ann Math Stat. 1947 Mar;18(1):50-60.

67. Benjamini Y, Hochberg Y. Controlling the False Discovery Rate: A Practical and Powerful Approach to Multiple Testing. J R Stat Soc Ser B Methodol. 1995;57(1):289-300. 
604

605

606

607

608

609

610

611

612

613

614

615

616

617

618

619

620

621

622

623

624

625

626

627

628

629

630

631

632

633

634

635

636

637

638

68. Dick GJ, Andersson AF, Baker BJ, Simmons SL, Thomas BC, Yelton AP, et al. Community-wide analysis of microbial genome sequence signatures. Genome Biol. 2009;10(8):R85.

69. Karlin S, Campbell AM, Mrazek J. Comparative DNA analysis across diverse genomes. Annu Rev Genet. 1998;32:185-225.

70. Wayne LG, Brenner DJ, Colwell RR, Grimont PAD, Kandler O, Krichevsky MI, et al. Report of the Ad Hoc Committee on Reconciliation of Approaches to Bacterial Systematics. Int J Syst Evol Microbiol. 1987;37(4):463-4.

71. Bohlin J, Snipen L, Hardy SP, Kristoffersen AB, Lagesen K, Donsvik T, et al. Analysis of intragenomic GC content homogeneity within prokaryotes. BMC Genomics. 2010 Aug 6;11:464.

72. Galtier N, Lobry JR. Relationships between genomic G+C content, RNA secondary structures, and optimal growth temperature in prokaryotes. J Mol Evol. 1997 Jun;44(6):632-6.

73. Wixon J. Featured organism: reductive evolution in bacteria: Buchnera sp., Rickettsia prowazekii and Mycobacterium leprae. Comp Funct Genomics. 2001;2(1):44-8.

74. Wu YW, Simmons BA, Singer SW. MaxBin 2.0: an automated binning algorithm to recover genomes from multiple metagenomic datasets. Bioinformatics. 2016 Feb 15;32(4):605-7.

75. Albertsen M, Hugenholtz P, Skarshewski A, Nielsen KL, Tyson GW, Nielsen PH. Genome sequences of rare, uncultured bacteria obtained by differential coverage binning of multiple metagenomes. Nat Biotechnol. 2013 Jun;31(6):533-8.

76. Imelfort M, Parks D, Woodcroft BJ, Dennis P, Hugenholtz P, Tyson GW. GroopM: an automated tool for the recovery of population genomes from related metagenomes. PeerJ. 2014;2:e603.

77. Pop M. Genome assembly reborn: recent computational challenges. Brief Bioinform. 2009 Jul;10(4):354-66.

78. Ghurye JS, Cepeda-Espinoza V, Pop M. Metagenomic Assembly: Overview, Challenges and Applications. Yale J Biol Med. 2016;89(3):353.

79. Frost LS, Leplae R, Summers AO, Toussaint A. Mobile genetic elements: the agents of open source evolution. Nat Rev Microbiol. 2005 Sep;3(9):722-32.

80. Hacker J, Kaper JB. Pathogenicity islands and the evolution of microbes. Annu Rev Microbiol. 2000;54:641-79.

81. Hurst LD, Merchant AR. High guanine-cytosine content is not an adaptation to high temperature: a comparative analysis amongst prokaryotes. Proc R Soc Lond B Biol Sci. 2001 Mar 7;268(1466):493-7.

82. Schattner P. Searching for RNA genes using base-composition statistics. Nucleic Acids Res. 2002 May 1;30(9):2076-82.

83. Daubin V, Lerat E, Perriere G. The source of laterally transferred genes in bacterial genomes. Genome Biol. 2003;4(9):R57. 
639 84. Garcia-Vallve S, Romeu A, Palau J. Horizontal gene transfer in bacterial and archaeal complete genomes. Genome Res. 2000 Nov;10(11):1719-25.

641 85. Rocha EPC, Danchin A. Base composition bias might result from competition for metabolic resources. Trends Genet. 2002 Jun;18(6):291-4.

86. Frank JA, Pan Y, Tooming-Klunderud A, Eijsink VGH, McHardy AC, Nederbragt AJ, et al. Improved metagenome assemblies and taxonomic binning using long-read circular consensus sequence data. Sci Rep. 2016 Jul;6(1):25373.

87. Bertrand D, Shaw J, Kalathiyappan M, Ng AHQ, Kumar MS, Li C, et al. Hybrid metagenomic assembly enables high-resolution analysis of resistance determinants and mobile elements in human microbiomes. Nat Biotechnol. 2019 Aug;37(8):937-44. 
Table $\mathbf{1}$ (on next page)

Reconstructed genome coverage and completeness 
1 Table 1 Reconstructed genome coverage and completeness

\begin{tabular}{|c|c|c|c|c|c|c|c|}
\hline Genome & $\begin{array}{l}\text { Genome NCBI } \\
\text { accessions }\end{array}$ & $\begin{array}{l}\text { MAG NCBI } \\
\text { accessions }\end{array}$ & MG Cov & $\% \mathrm{CR}^{\mathrm{b}}$ & $\mathrm{NR}^{\mathrm{c}}$ & $\begin{array}{l}\text { mean NR } \\
\text { length (bp) }\end{array}$ & $\begin{array}{l}\text { NR length } \\
\text { range }\end{array}$ \\
\hline \multirow[t]{2}{*}{ HL-46 } & EI34DRAFT_7210 & GCA_001314525.1 & $3.9 x$ & $40 \%$ & 284 & 4742 & $1007 . .42318$ \\
\hline & EI34DRAFT_6181 ${ }^{\mathrm{d}}$ & & $3.9 x$ & $25 \%$ & 7 & 18136 & $1108 . .49149$ \\
\hline HL-48 & CY41DRAFT & GCA_001314875.1 & $69 x$ & $95 \%$ & 29 & 1892 & $330 . .53737$ \\
\hline HL-49 & K302DRAFT & GCA_001314815.1 & $9.7 x$ & $91 \%$ & 89 & 3234 & $209 . .25366$ \\
\hline HL-53 & Ga0003345 & GCA_001314555.1 & $113 x$ & $98 \%$ & 15 & 1564 & $952 . .6133$ \\
\hline HL-55 & K417DRAFT & GCA_001314845.1 & $11 \mathrm{x}$ & $95 \%$ & 34 & 3574 & $417 . .45387$ \\
\hline HL-58 & CD01DRAFT & GCA_001314605.1 & $128 \mathrm{x}$ & $99 \%$ & 13 & 1124 & $959 . .12996$ \\
\hline \multirow[t]{4}{*}{ HL-91 } & Ga0058931_14 & GCA_001314645.1 & $226 x$ & $97 \%$ & 20 & 3129 & $135 . .11341$ \\
\hline & Ga0058931_11 ${ }^{\mathrm{d}}$ & & $227 x$ & $97 \%$ & 6 & 2188 & $914 . .4391$ \\
\hline & Ga0058931_13 ${ }^{\mathrm{d}}$ & & $158 \mathrm{x}$ & $0 \%$ & 1 & 113349 & 113349 \\
\hline & Ga0058931_12d & & $160 x$ & $0 \%$ & 1 & 97917 & 97917 \\
\hline HL-93 & $\mathrm{Ga} 0071314$ & GCA_001314745.1 & $11 x$ & $85 \%$ & 98 & 3605 & $232 . .78515$ \\
\hline \multirow[t]{3}{*}{ HL-109 } & Ga0071312_11 & GCA_001314785.1 & $612 x$ & $87 \%$ & 20 & 1835 & $204 . .63971$ \\
\hline & Ga0071312_12 & & $669 x$ & $92 \%$ & 28 & 1285 & $506 . .52589$ \\
\hline & $\mathrm{Ga} 0071312-_{13}^{\mathrm{d}}$ & & $615 x$ & $95 \%$ & 3 & 6053 & $1908 . .10088$ \\
\hline HL-111 & $\mathrm{Ga} 0071316$ & GCA_001314765.1 & $18 \mathrm{x}$ & $95 \%$ & 39 & 1589 & $501 . .20407$ \\
\hline
\end{tabular}

2 a Metagenomic read coverage

3 b Percentage of the genome represented in the MAG

$4{ }^{\mathrm{c}}$ Number of not-binned regions

5 d Predicted to be an extrachromosomal element

6 
Table 2 (on next page)

$\% \mathrm{G}+\mathrm{C}$ analysis 
1 Table 2. Comparison of $\% \mathrm{G}+\mathrm{C}$ for genomes, $\mathrm{CRs}$ and NRs

2

\begin{tabular}{ll|r|rrr|rrr}
\hline & & Genome & \multicolumn{3}{|c}{ CRs } & \multicolumn{3}{c}{ NRs } \\
\cline { 3 - 8 } & molecule & mean & mean & distance & p-value & mean & distance & p-value \\
\hline HL-46 & EI34DRAFT_7210 & 64.42 & $63.96 \pm 1.94$ & $1.55 \pm 1.25$ & 0.997 & $65.12 \pm 2.13$ & $1.61 \pm 1.56$ & $0 . .263$ \\
HL-46 & EI34DRAFT_6181 & 59.94 & $60.78 \pm 2.27$ & $1.97 \pm 1.41$ & 0.856 & $60.97 \pm 1.78$ & $1.92 \pm 0.75$ & 0.605 \\
\hline HL-48 & CY41DRAFT & 58.98 & $59.00 \pm 1.52$ & $1.01 \pm 1.13$ & 0.996 & $45.76 \pm 19.69$ & $13.22 \pm 19.69$ & $<\mathbf{0 . 0 0 1}$ \\
HL-49 & K302DRAFT & 42.22 & $42.24 \pm 1.71$ & $1.15 \pm 1.27$ & 0.434 & $42.73 \pm 3.37$ & $2.44 \pm 2.38$ & $\mathbf{0 . 0 0 1}$ \\
HL-53 & Ga0003345 & 47.50 & $46.95 \pm 1.61$ & $0.96 \pm 1.40$ & 0.031 & $48.83 \pm 3.55$ & $3.70 \pm 0.82$ & $<\mathbf{0 . 0 0 1}$ \\
HL-55 & K417DRAFT & 56.26 & $55.87 \pm 1.97$ & $1.42 \pm 1.41$ & 0.025 & $55.44 \pm 3.30$ & $3.00 \pm 1.59$ & $\mathbf{0 . 0 0 1}$ \\
HL-58 & CD01DRAFT & 57.56 & $56.83 \pm 2.61$ & $1.69 \pm 2.12$ & 0.047 & $56.11 \pm 3.69$ & $3.93 \pm 0.51$ & 0.016 \\
HL-91 & Ga0058931_11 & 61.75 & $62.05 \pm 0.25$ & $0.31 \pm 0.23$ & 0.954 & $60.39 \pm 3.17$ & $2.79 \pm 2.02$ & 0.053 \\
HL-91 & Ga0058931_12 & 60.37 & nd & nd & nd & nd & nd & nd \\
HL-91 & Ga0058931_13 & 61.77 & nd & nd & nd & nd & nd & nd \\
HL-91 & Ga0058931_14 & 61.84 & $60.99 \pm 1.90$ & $1.33 \pm 1.60$ & 0.030 & $59.11 \pm 2.96$ & $3.52 \pm 1.96$ & $\mathbf{0 . 0 0 5}$ \\
\hline HL-93 & Ga0071314_11 & 55.88 & $56.75 \pm 2.20$ & $1.75 \pm 1.59$ & 1.000 & $56.08 \pm 4.42$ & $3.6 \pm 2.57$ & $<\mathbf{0 . 0 0 1}$ \\
HL-109 & Ga0071312_11 & 64.09 & $64.55 \pm 1.46$ & $1.12 \pm 1.05$ & 0.715 & $60.96 \pm 3.02$ & $3.28 \pm 2.85$ & 0.073 \\
HL-109 & Ga0071312_12 & 64.07 & $63.89 \pm 1.41$ & $0.92 \pm 1.09$ & 0.169 & $63.11 \pm 2.21$ & $1.94 \pm 1.43$ & 0.593 \\
HL-109 & Ga0071312_13 & 65.34 & $65.47 \pm 0.07$ & $0.13 \pm 0.07$ & 0.778 & $61.68 \pm 2.24$ & $3.66 \pm 2.24$ & 0.009 \\
HL-111 & Ga0071316_11 & 68.12 & $68.20 \pm 1.44$ & $0.99 \pm 1.05$ & 0.465 & $64.26 \pm 1.39$ & $3.86 \pm 1.39$ & $<\mathbf{0 . 0 0 1}$ \\
\hline
\end{tabular}

$3 \quad{ }^{a}$ Bold type indicates significant results $(\mathrm{P} \leq 0.005)$.

4 b Not determined because the entire molecule was missing from the reconstructed genome. 
Table 3 (on next page)

Tetranucleotide frequency analysis 
1 Table 3. Tetranucleotide frequency $\chi^{2}$ analysis.

\begin{tabular}{ll|ccc|ccr}
\hline & & \multicolumn{3}{|c|}{ CR } & \multicolumn{3}{c}{ NR } \\
\hline & molecule & mean & sd & p-value & mean & sd & p-value \\
\hline HL-46 & EI34DRAFT_6181 & 0.2323 & 0.1883 & 0.154 & 0.1518 & 0.1429 & 0.983 \\
HL-46 & EI34DRAFT_7210 & 0.2042 & 0.0696 & 0.896 & 0.1701 & 0.1332 & 0.975 \\
HL-48 & CY41DRAFT & 0.0276 & 0.0577 & 0.387 & 0.4425 & 0.2689 & $<\mathbf{0 . 0 0 1}$ \\
HL-49 & K302DRAFT & 0.0522 & 0.0431 & 0.757 & 0.2340 & 0.2164 & $<\mathbf{0 . 0 0 1}$ \\
HL-53 & Ga0003345 & 0.0261 & 0.0451 & $\mathbf{0 . 0 0 1}$ & 0.3851 & 0.1525 & $<\mathbf{0 . 0 0 1}$ \\
HL-55 & K417DRAFT & 0.0458 & 0.0726 & 0.086 & 0.2774 & 0.2168 & $\mathbf{0 . 0 0 4}$ \\
HL-58 & CD01DRAFT & 0.0761 & 0.1451 & 0.008 & 0.2974 & 0.969 & $\mathbf{0 . 0 0 4}$ \\
HL-91 & Ga0058931_11 & 0.0266 & 0.0213 & 0.313 & 0.3043 & 0.1416 & 0.011 \\
HL-91 & Ga0058931_12 & nd & nd & nd & nd & nd & nd \\
HL-91 & Ga0058931_13 & nd & nd & nd & nd & nd & nd \\
HL-91 & Ga0058931_14 & 0.0557 & 0.0647 & $\mathbf{0 . 0 0 4}$ & 0.3614 & 0.2052 & $<\mathbf{0 . 0 0 1}$ \\
HL-93 & Ga0071314_11 & 0.0925 & 0.0738 & 0.993 & 0.2254 & 0.1595 & 0.062 \\
HL-109 & Ga0071312_11 & 0.0262 & 0.0401 & 0.396 & 0.3148 & 0.1842 & 0.087 \\
HL-109 & Ga0071312_12 & 0.0216 & 0.0281 & 0.076 & 0.2907 & 0.1913 & 0.231 \\
HL-109 & Ga0071312_13 & 0.0048 & 0.0019 & 0.538 & 0.3651 & 0.2299 & 0.016 \\
HL-111 & Ga0071316_11 & 0.0396 & 0.0561 & 0.322 & 0.4504 & 0.1640 & $<\mathbf{0 . 0 0 1}$ \\
\hline
\end{tabular}

2 a Bold text indicates significant result

3 b Not determined because the entire molecule was missing from the reconstructed genome. 
Table 4 (on next page)

Genomic redundancy 
1 Table 4. Metagenomic redundancy.

\begin{tabular}{ll|r|rcr|rrr}
\hline & & $\begin{array}{r}\text { Genome } \\
\text { mean }\end{array}$ & mean & $\begin{array}{c}\text { CR } \\
\text { distance }\end{array}$ & p-value & mean & NR \\
distance & p-value \\
HL-46 & EI34DRAFT_6181 & 2.76 & 2.78 & $0.26 \pm 0.15$ & 0.992 & 2.42 & $0.43 \pm 0.31$ & 0.264 \\
HL-46 & EI34DRAFT_7210 & 5.98 & 4.43 & $2.95 \pm 2.34$ & 0.978 & 4.99 & $4.01 \pm 13.35$ & 0.649 \\
HL-48 & CY41DRAFT & 72.40 & 69.29 & $3.65 \pm 2.45$ & 1.000 & 140.93 & $100.97 \pm 153.33$ & $<\mathbf{0 . 0 0 1}$ \\
\hline HL-49 & K302DRAFT & 8.97 & 8.67 & $0.51 \pm 0.58$ & 0.999 & 11.38 & $4.16 \pm 17.52$ & $\mathbf{0 . 0 0 2}$ \\
HL-53 & Ga0003345 & 441.81 & 446.29 & $24.51 \pm 18.21$ & 0.073 & 517.07 & $115.56 \pm 59.71$ & $<\mathbf{0 . 0 0 1}$ \\
HL-55 & K417DRAFT & 16.76 & 15.35 & $7.06 \pm 10.45$ & 0.679 & 117.37 & $110.35 \pm 333.81$ & $<\mathbf{0 . 0 0 1}$ \\
HL-58 & CD01DRAFT & 128.28 & 127.85 & $9.10 \pm 15.71$ & 1.000 & 180.14 & $60.44 \pm 27.54$ & $<\mathbf{0 . 0 0 1}$ \\
HL-91 & Ga0058931_11 & 231.39 & 228.46 & $3.64 \pm 2.25$ & 0.786 & 311.6 & $91.27 \pm 97.44$ & $\mathbf{0 . 0 0 1}$ \\
HL-91 & Ga0058931_12 & 163.24 & nd ${ }^{\mathrm{b}}$ & $\mathrm{nd}$ & $\mathrm{nd}$ & $\mathrm{nd}$ & $\mathrm{nd}$ & nd \\
HL-91 & Ga0058931_13 & 168.27 & $\mathrm{nd}$ & $\mathrm{nd}$ & $\mathrm{nd}$ & $\mathrm{nd}$ & $\mathrm{nd}$ & $\mathrm{nd}$ \\
HL-91 & Ga0058931_14 & 227.56 & 231.77 & $8.18 \pm 6.59$ & 0.220 & 273.03 & $97.82 \pm 117.47$ & $<\mathbf{0 . 0 0 1}$ \\
HL-93 & Ga0071314_11 & 50.87 & 50.03 & $4.04 \pm 2.92$ & 1.000 & 65.73 & $16.16 \pm 35.87$ & $<\mathbf{0 . 0 0 1}$ \\
\hline HL-109 & Ga0071312_11 & 3103.11 & 3098.73 & $97.47 \pm 72.15$ & 0.748 & 3072.59 & $323.24 \pm 240.86$ & $\mathbf{0 . 0 0 5}$ \\
HL-109 & Ga0071312_12 & 2821.18 & 2822.26 & $113.08 \pm 78.18$ & 0.124 & 2778.03 & $352.81 \pm 436.28$ & $\mathbf{0 . 0 0 3}$ \\
HL-109 & Ga0071312_13 & 2853.84 & 2901.40 & $47.56 \pm 9.73$ & 0.179 & 2097.01 & $756.83 \pm 256.91$ & 0.018 \\
HL-111 & Ga0071316_11 & 90.14 & 88.03 & $3.98 \pm 4.31$ & 0.993 & 98.25 & $38.42 \pm 104.87$ & 0.027 \\
\hline
\end{tabular}

2 a Bold text indicates significant result

3 b Not determined because the entire molecule was missing from the reconstructed genome. 
Figure 1

Distributions of $\% \mathrm{G}+\mathrm{C}$ for MDR and CDR genomic regions.

$\mathrm{G}+\mathrm{C}$ composition was determined for individual regions identified as CDRs or MDRs. Bar

height represents the percentage of regions in the category. Black bars, CDRs; white bars, MDRs. 


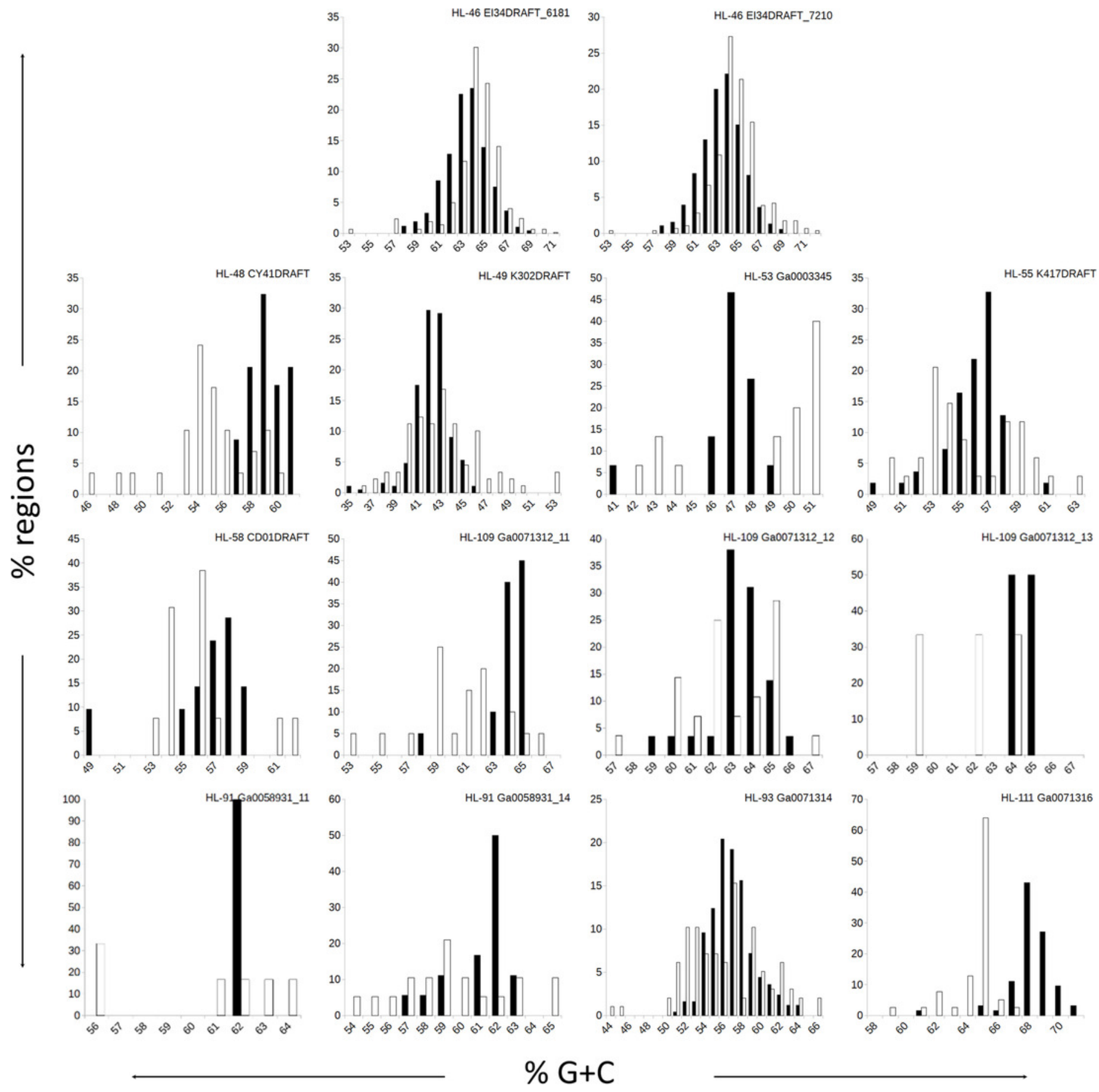




\section{Figure 2}

Analysis of HL-111 genome.

Ring 1 (outermost, black) - genome sequence; ring 2 (grey bars) - missed detection regions (MDRs); ring 3 (teal) - tetranucleotide frequency (TNF) distance $\chi^{2}$ values; ring 4 (orange) $\% \mathrm{G}+\mathrm{C}$; ring 5 (blue) - intragenome redundancy; ring 6 (magenta) - metagenome redundancy. Values were calculated across $2000 \mathrm{nt}$ windows with a step size of $1000 \mathrm{nt}$. For TNF, $\chi^{2}$ was calculated for the windows using the whole molecule frequencies as the expected. Data for other genomes analyzed is presented in Figure S1. Circlular plots were generated using Circos v0.69.3 ( Krzywinski, Schein et al. 2009 ) . 
Outermost to innermost

1. Scale in $\mathrm{Mb}$

2. Genome

3. Not-binned regions

4. Tetranucleotide chi square

5. $\% \mathrm{G}+\mathrm{C}$

6. Intragenome repetitiveness

7. Metagenome repetitiveness

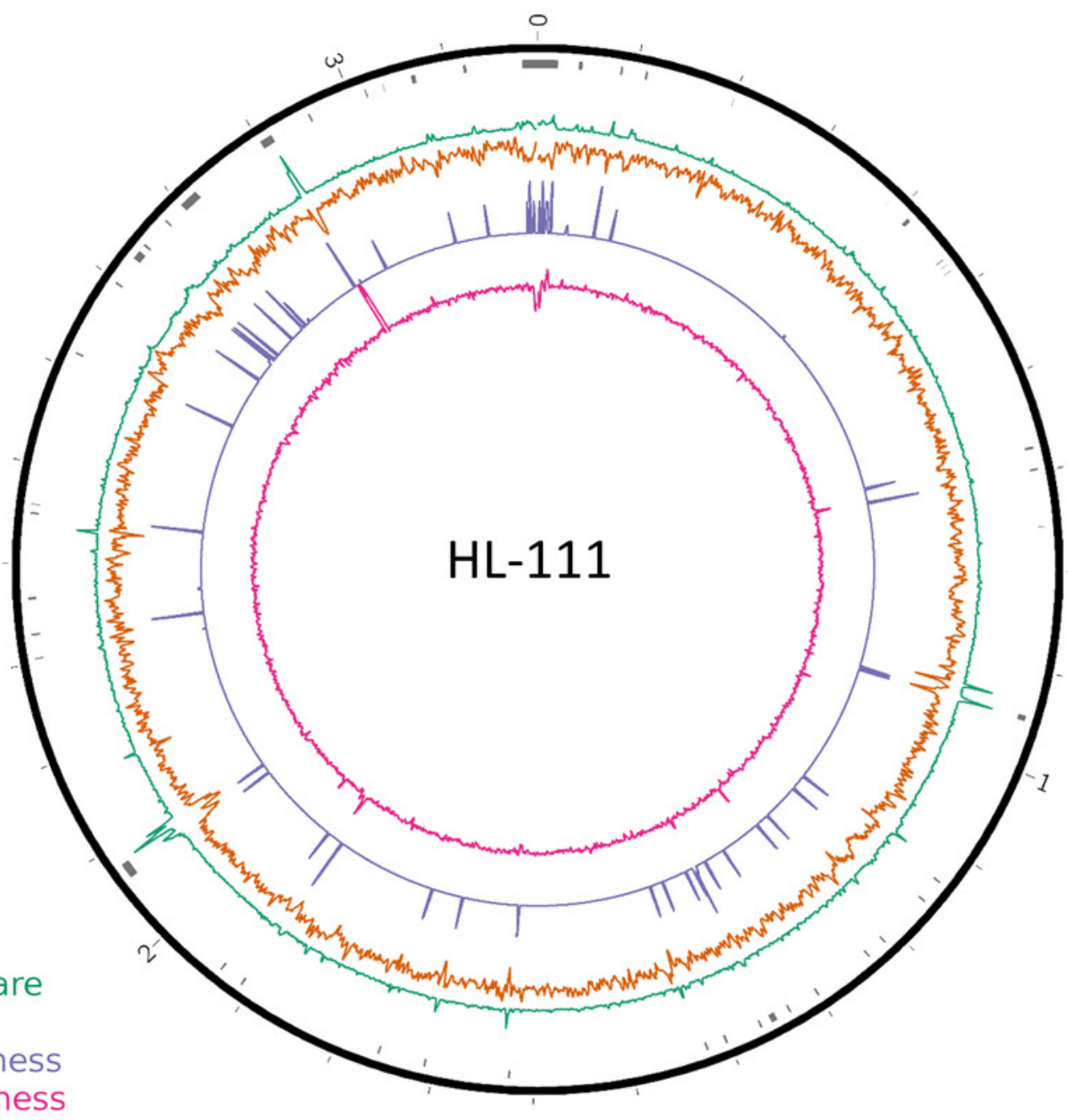

Ga0071316 11 


\section{Figure 3}

Repeat content of genomes versus MAGs

Box plot representation of the total fraction of each genome/MAG in a repeat region as determined by NUCmer ( $\geq 97 \%$ identity; center line, median; box limits, upper and lower quartiles; whiskers, 1.5xinterquartile range; diamonds, outliers). UCC MAG and genome comparison were significantly different ( $p=0.01$; Mann-Whitney U). 


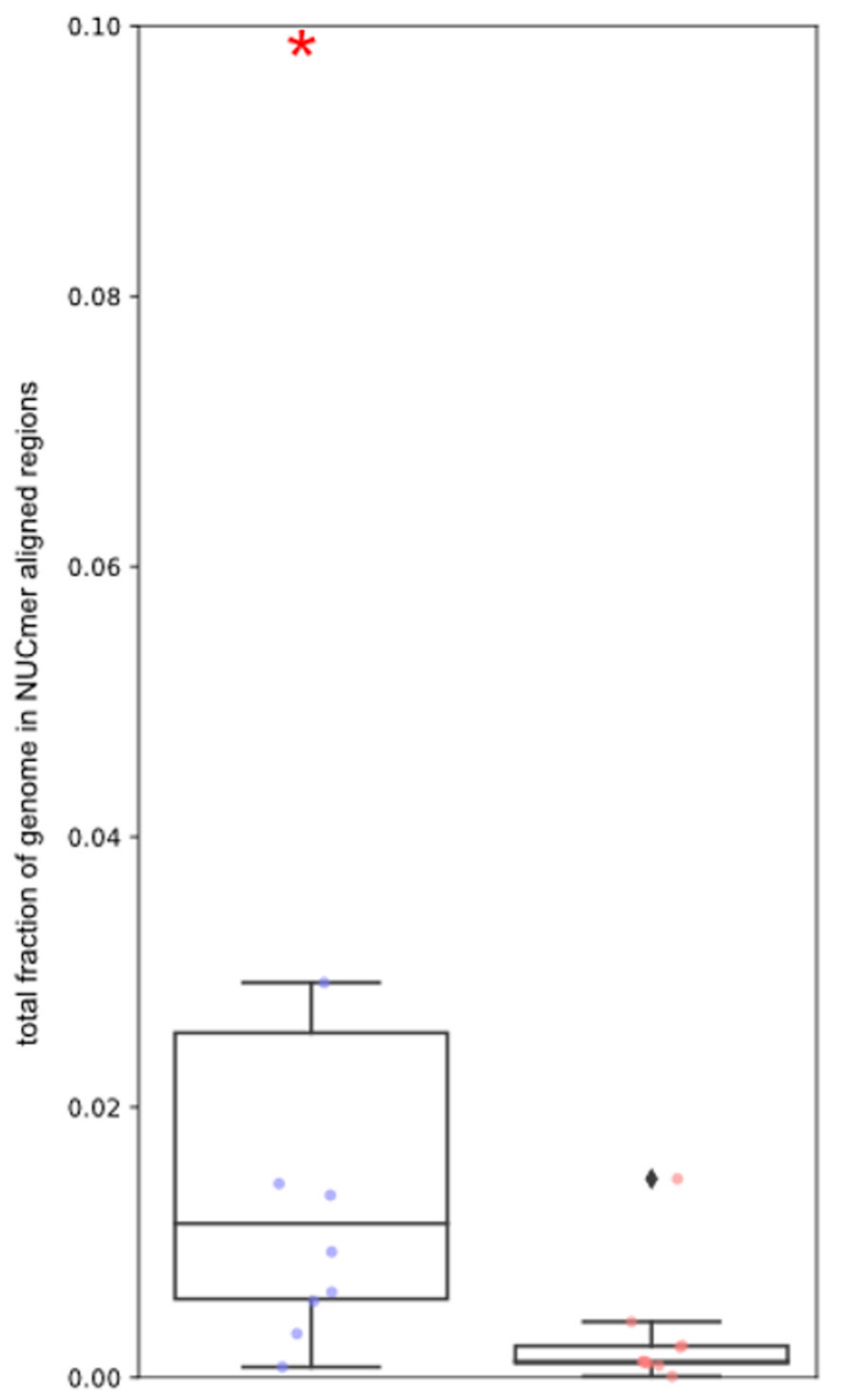

Hot Lake Genomes

( $n=10$ )
Hot Lake MAGs

$$
(\mathrm{n}=10)
$$




\section{Figure 4}

Functional categorization of genes present on MDRs.

The gene features of each genome region were assigned to functional COG categories or as non-coding genes (rRNA; tRNA; ncRNA). Organisms' gene sets were compared using Principal Component Analysis. Organisms are represented by colors (HL-46, yellow; HL-48, purple; HL-49, blue; HL-53, light blue; HL-55, gray; HL-58, orange; HL-91, black; HL-93, pink; HL-109, red; HL-111, green). The genome region categories are represented by shapes (whole isolate genomes, circles; CDRs, squares; MDRs, triangles; extrachromosomal elements, diamonds). COG categories: $A$ - RNA processing and modification; $B$ - Chromatic structure and dynamics; $C$ - Energy production and conversion; D - Cell cycle control, cell division, chromosome partitioning; $E$ - Amino acid transport and metabolism; $F$ - Nucleotide transport and metabolism; $G$ - Carbohydrate transport and metabolism; $H$ - Coenzyme transport and metabolism; I - Lipid transport and metabolism; J - Translation, ribosomal structure and biogenesis; $K$ - Transcription; $L$ - DNA replication, recombination and repair; $M$ - Cell wall/membrane/envelope biogenesis; $N$ - Cell motility; $O$ - Post-translational modification, protein turnover, chaperones; $P$ - Inorganic ion transport and metabolism; $Q$ - Secondary metabolites biosynthesis, transport and catabolism; $R$ - General function prediction; $S$ Function unknown; $T$ - Signal transduction mechanisms; $U$ - Intracellular trafficking, secretion and vesicular transport; $V$ - Defense mechanisms; $W$ - Extracellular structures; $X$ - Mobilome, transposons, phages; $Y$ - Nuclear structure; $Z$ - Cytoskeleton. 


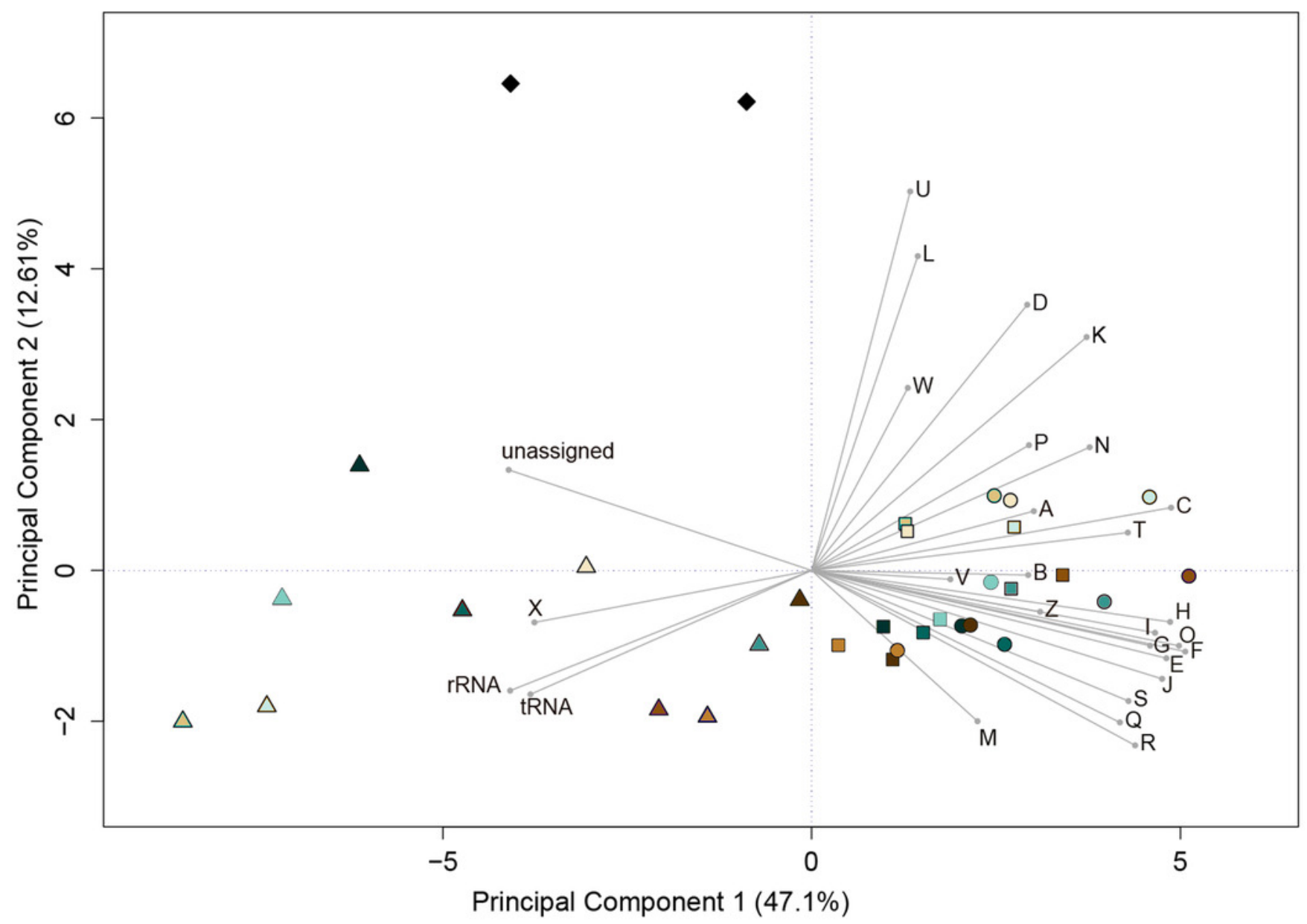

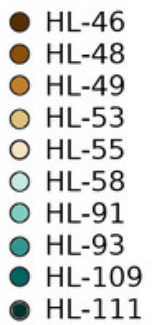

- Whole isolate genome

- Correctly-binned regions

A Not-binned regions

- Extra-chromosomal elements

- HL-111 


\section{Figure 5}

Tara Ocean MAG nucleotide composition analysis

(A) $\% G+C$ variance analysis. Box plot representation of the $\% G+C$ variance for each $2,000 \mathrm{bp}$ segment of genome/MAG (sliding window step: $500 \mathrm{bp}$; center line, median; box limits, upper and lower quartiles; whiskers, $1.5 \times$ interquartile range; diamonds, outliers). Comparisons between Tara Oceans MAG datasets and RefSeq genomes were significantly different $(p<$ 0.001; Mann-Whitney U with Benjamini-Hochberg False Discovery Rate Correction (BH FDR)). (B) Tetranucleotide analysis. Box plot representaiton of the variance in Pearson correlation values of the tetranucleotide Z-scores for a pair-wise comparison of each $10 \mathrm{~kb}$ segment of genome/MAG (sliding window step: 5kb; center line, median; box limits, upper and lower quartiles; whiskers 1.5x interquartile range; diamonds, outliers). Comparisons between Tara Oceans MAG datasets and RefSeq genomes were significantly different ( $p<0.001$; MannWhitney U with BH FDR Correction). Red asterisks denote the existence of outliers outside of the displayed range. 

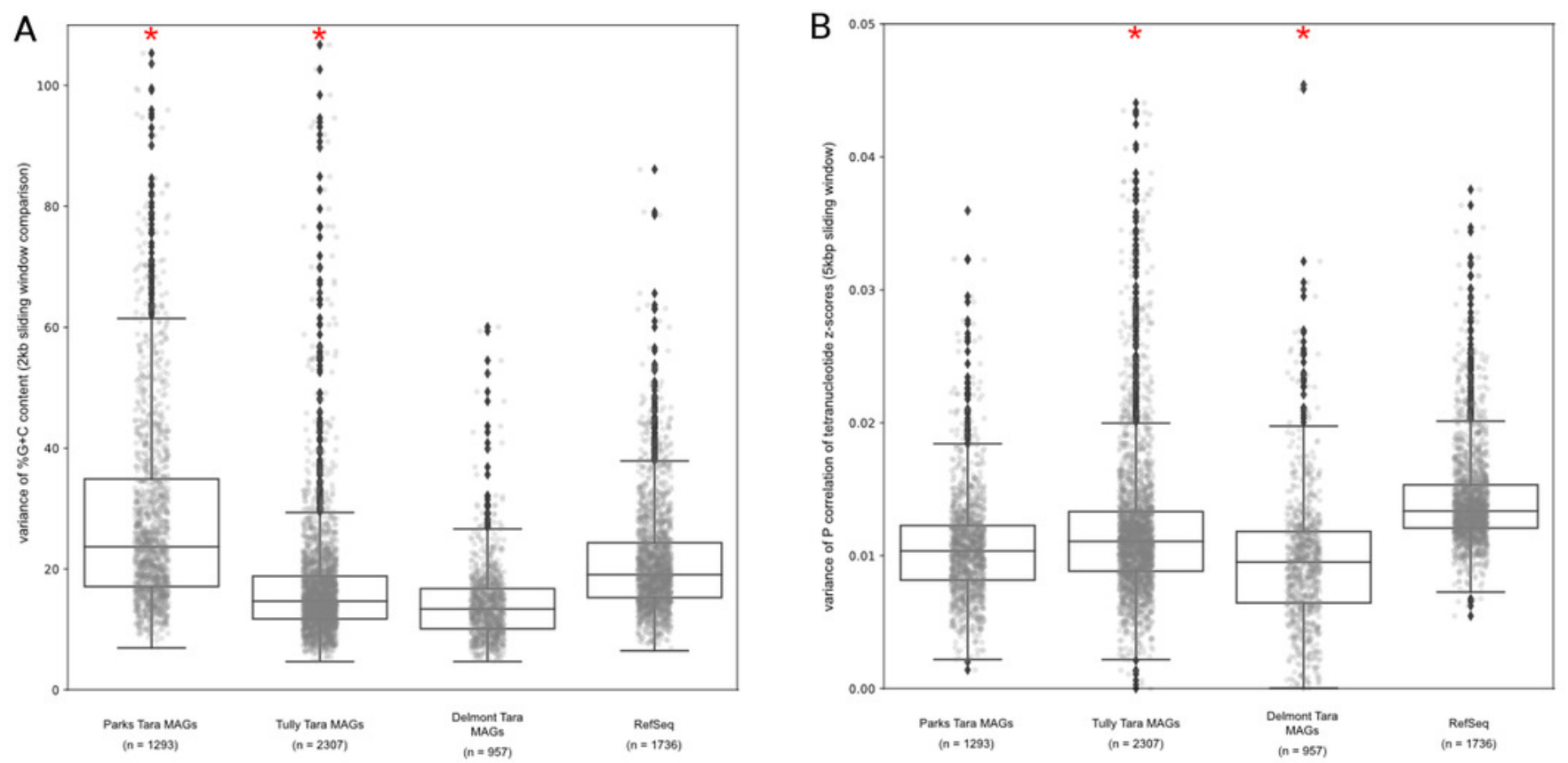


\section{Figure 6}

\section{Tara Ocean MAG repeat content}

Box plot representation of the total fraction of each genome/MAG in a repeat region as determined by NUCmer ( $\geq 97 \%$ identity; center line, median; box limits, upper and lower quartiles; whiskers, $1.5 \times$ interquartile range; diamonds, outliers). Comparisons between Tara Oceans MAG datasets and RefSeq genomes were significantly different $(p<0.001$; MannWhitney U with BH FDR Correction). Red asterisks denote the existence of outliers outside of the displayed dataset. 


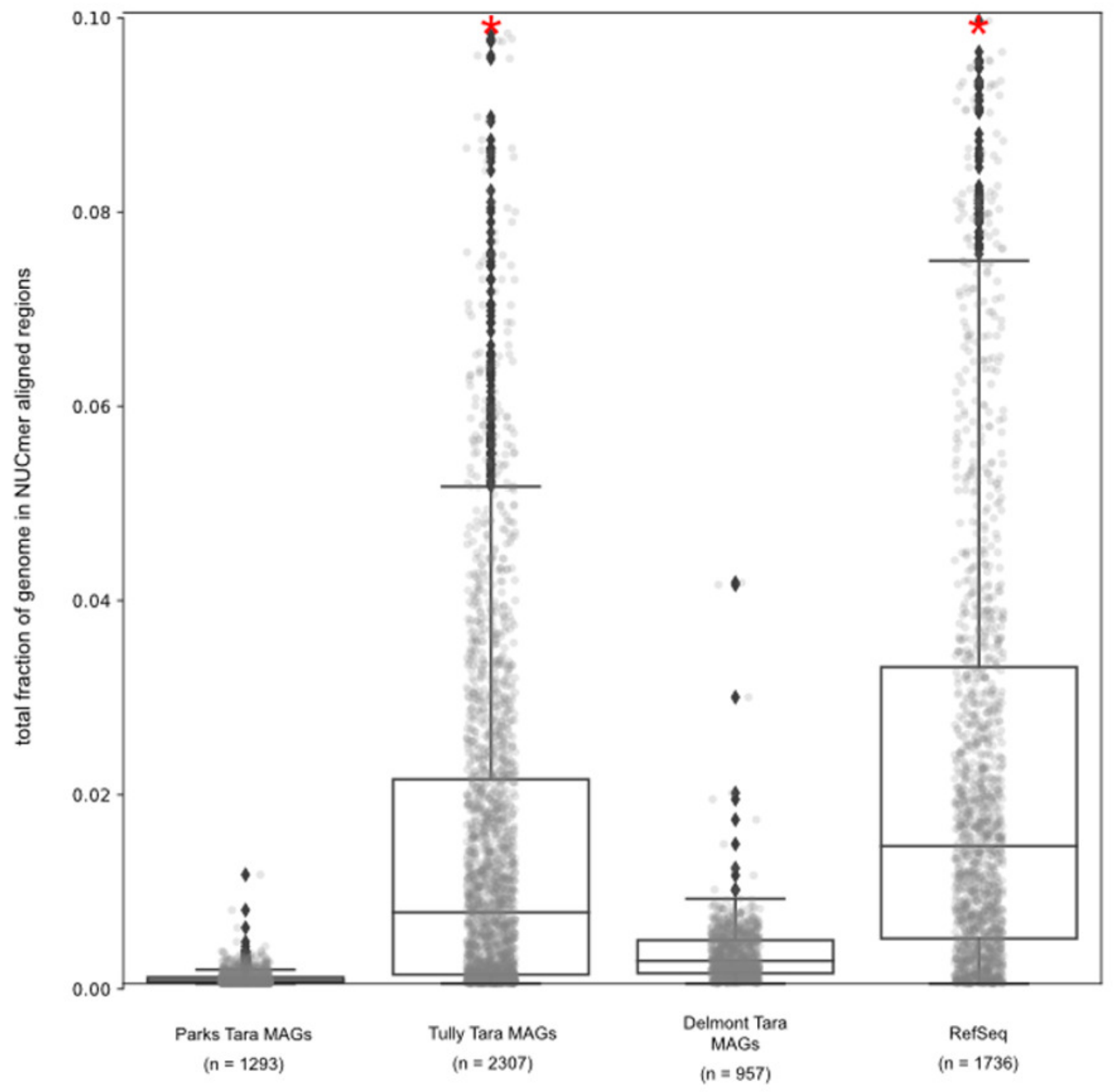

